Classification

Physics Abstracts

$61.16 \mathrm{D}-61.16 \mathrm{~F}-06.00$

\title{
Quantitative in situ experiments and possible artifacts
}

\author{
J. Pelissier $\left({ }^{1}\right)$ and F. Louchet $\left({ }^{2}\right)$ \\ ( $\left.{ }^{1}\right)$ CEA-CEREM, Centre d'Etudes Nucléaires de Grenoble, BP 85X, 38041 Grenoble Cedex, France \\ $\left.{ }^{2}\right)$ LTPCM, ENSEEG, INP de Grenoble, BP 75, 38402 st Martin d'Heres Cedex, France
}

(Received January 18, 1993; accepted February 26, 1993)

\begin{abstract}
Résumé. - Quelques techniques spécifiques destinées à tirer des expériences in situ des informations fiables et quantitatives et à éviter la plupart des artefacts dûs aux surfaces des lames minces et aux dommages d'irradiation sont discutées et illustrées sur quelques exemples. En particulier des mesures de la charge appliquée, de la contrainte et de la déformation locales, de la densité de dislocations mobiles ainsi que la détermination des énergies d'activation reliées au mouvement des dislocations sont examinées.
\end{abstract}

\begin{abstract}
Some specific techniques to be used to obtain reliable quantitative information from in situ straining experiments and to avoid most artifacts due to thin foil surfaces and to radiation damage are discussed and illustrated on some examples. In particular measurements of external load, local stress and strain, mobile dislocation densities and determination of activation energies related to dislocation motion are envisaged.
\end{abstract}

\section{Introduction.}

In situ experiments on crystalline materials and in particular straining experiments are known to give fascinating dynamic images of moving dislocations. Interesting informations can be obtained from these records regarding the types of mechanisms responsible of the plastic deformation. However, valuable quantitative information can be drawn from these experiments using some specific techniques. The aim of the present paper is to discuss the techniques to be used to get reliable quantitative information and to avoid as far as possible the different possible artifacts due either to the foil surfaces or to the electron beam.

\section{Temperature measurements.}

In a high temperature straining stage, temperature is usually measured by a thermocouple. A careful calibration is often necessary, due to the small dimensions of the system which does not always ensure a good homogeneity of the temperature. This calibration can be achieved for instance using microcells deposited on a foil and containing low melting point metals or eutectics 
(e.g. Sn, Al-Ge) (Fig. 1). A carbon support is first deposited on the foil (Fig. 2a). The metal is then deposited by vacuum evaporation through a grid (Fig. 2b). A carbon film coating prevents evaporation in the microscope (Fig. 2c). This method allows a temperature calibration with an accuracy of about $10^{\circ} \mathrm{C}$.

\section{TEMPERATURE MEASUREMENTS CONTROL}

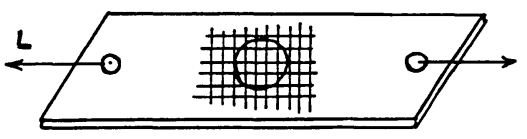

Fig. 1. - Microcell network deposited on a foil, used for temperature calibration of a heating stage. Each cell contains a low melting point alloy.

Material deposition microcells by vacuum evaporation

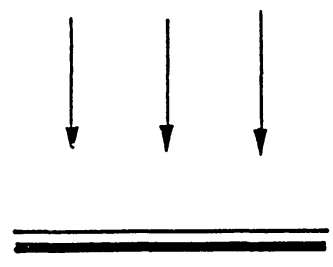

a

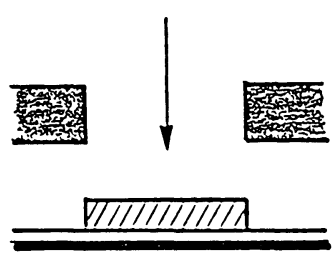

b
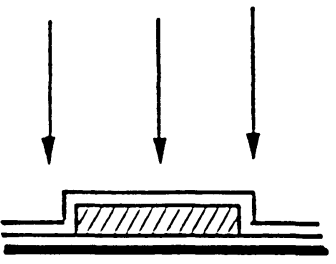

C

Fig. 2. - Three steps of cell deposition (see text).

\section{Load measurements.}

The external load applied to the specimen is nearly impossible to relate to the local stress in the observed regions of the specimen, due to non uniform thickness of the foil and to the often irregular shape of the hole. The particular methods for estimating the local stress are discussed in the next paragraph. Nevertheless, the measurement of the external load applied to the specimen can be of interest either to bring looseness down to zero at the beginning of the experiment, or to keep the load constant during heating (differential expansions) and during tilting (e.g. double tilt heating-straining stage, [1]). In this case, the total strain is measured by a strain gauge (Fig. 3). The measured value is compared to upper and lower required values displayed on two operational amplifiers. As soon as the measured value lies out of the imposed interval, a signal is sent by one of the two amplifiers to the hydraulic power unit which adjusts the strain back into the right interval. 


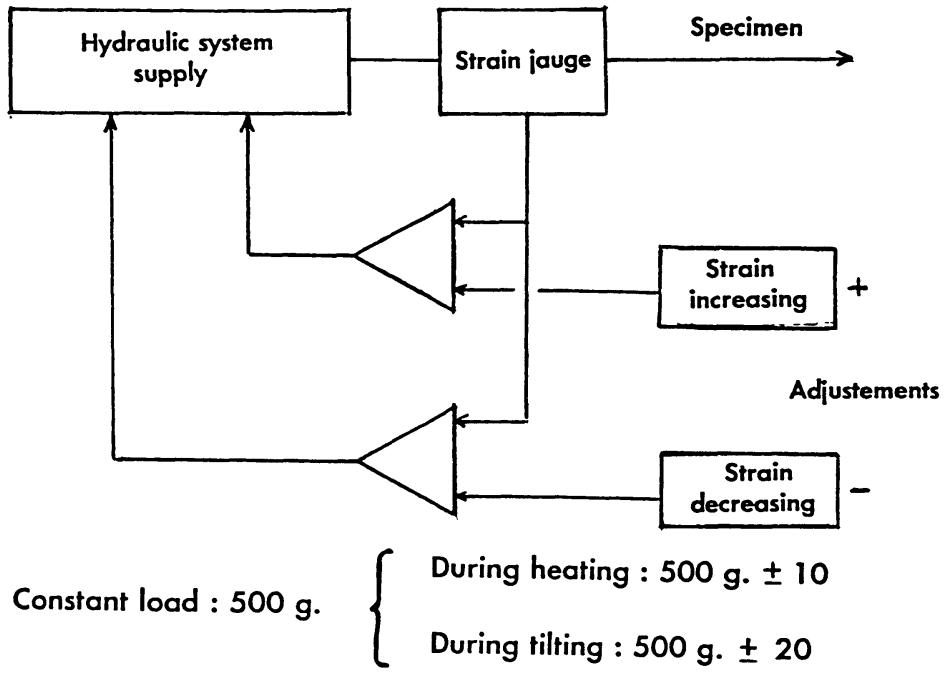

Fig. 3. - Control of the applied load (see text).

\section{Stress measurements.}

One of the main problems of in situ straining experiments is to estimate the stress in a thin foil. The specimen has usually a rectangular shape, with a hole at the center. Finite elements calculations of stress around a circular hole [2] show, as expected intuitively, that the maximum uniaxial stress is found at the sides of the hole parallel to the applied load. In real life however, holes are far from being circular. Intuition and experience are the best means of finding regions where deformation is likely to take place, and stress estimates are usually performed using dislocation curvatures.

In materials without lattice friction, curvature radii of dislocations at equilibrium scale as the reciprocal local resolved shear stress

$$
\tau=\mu b / R
$$

and a measurement of $R$, taking into account the geometrical correction due to the projection of the slip plane on the observation plane, gives a reasonable estimate of $\tau$.

In the case of materials with lattice friction, loops are far from being circular, but stress can be estimated from local curvatures. In BCC metals at low temperatures, curvatures radii of non screw segments trailing screw dipoles are related to stress by equation (1). In diamond cubic crystals, an elastic calculation has been performed [3] which shows that the stress-curvature relation is different according whether the bends connect two $60^{\circ}$ dislocations or a $60^{\circ}$ dislocation and a screw dislocation. Local stresses can be estimated from the diagrams given in [3].

\section{Strain measurements.}

As shown in figure 4 the total strain at the end of the experiment can be measured from the final shape of an initially circular hole obtained by ion milling. In the case of localized slip, the local strain can be reached using either local shear at slip traces (Fig. 5), or step heights $h$ at the hole 


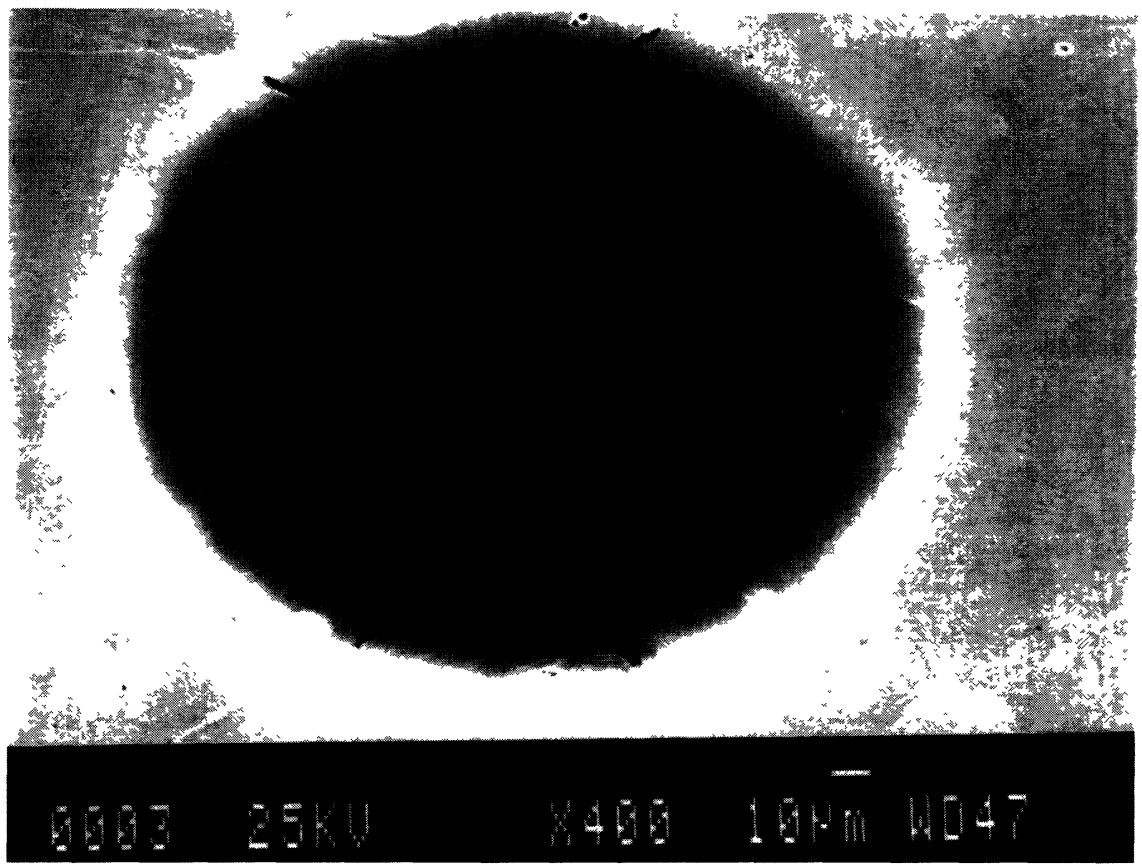

Fig. 4. - A Ge foil thinned by ion milling. The initial shape of the hole was circular. The final strain is directly measured from the hole ellipticity.

side. The number $n$ of dislocations scanning a given area on a single slip plane also gives an estimate of the local strain $\varepsilon=n b / b=n$.

\section{Dislocation velocities and activation energies.}

In the case where dislocations move smoothly by a double-kink mechanism, velocities can be easily measured through a frame by frame analysis of the video records. Measurements at different temperatures give directly activation energies using Arrhenius plots. In the case where a second order friction is experienced by kinks (diamond cubic structures), two activation energies have to be determined, corresponding resp. to double kink formation and to kink migration. Since kinks have here a finite mean free path, dislocation velocities depend on their lengths, and a measurement of this length dependence allows a complete determination of activation energies [4]. Sometimes, dislocation motion is not smooth but jerky. In some cases (HCP metals, intermetallics), this behaviour is ascribed to a locking-unlocking mechanism, and activation parameters are also obtainable by measuring flight times and waiting time [5].

When dislocation motion is no more controlled by a double kink mechanism, glide is controlled by localized obstacles. Waiting times $\delta t$ in front of these obstacles can be measured and are related to activation energies by the classical relation:

$$
1 / \delta t=\nu_{\mathrm{D}}(b / l) \exp (-\Delta G(\tau) / k T)
$$

where $\nu_{\mathrm{D}}$ is the Debye frequency, and $l$ the length of the vibrating segment. The total activation energy $\Delta G_{0}$ can then be calculated by:

$$
\Delta G_{0}=\Delta G(\tau)+l b^{2} \tau
$$




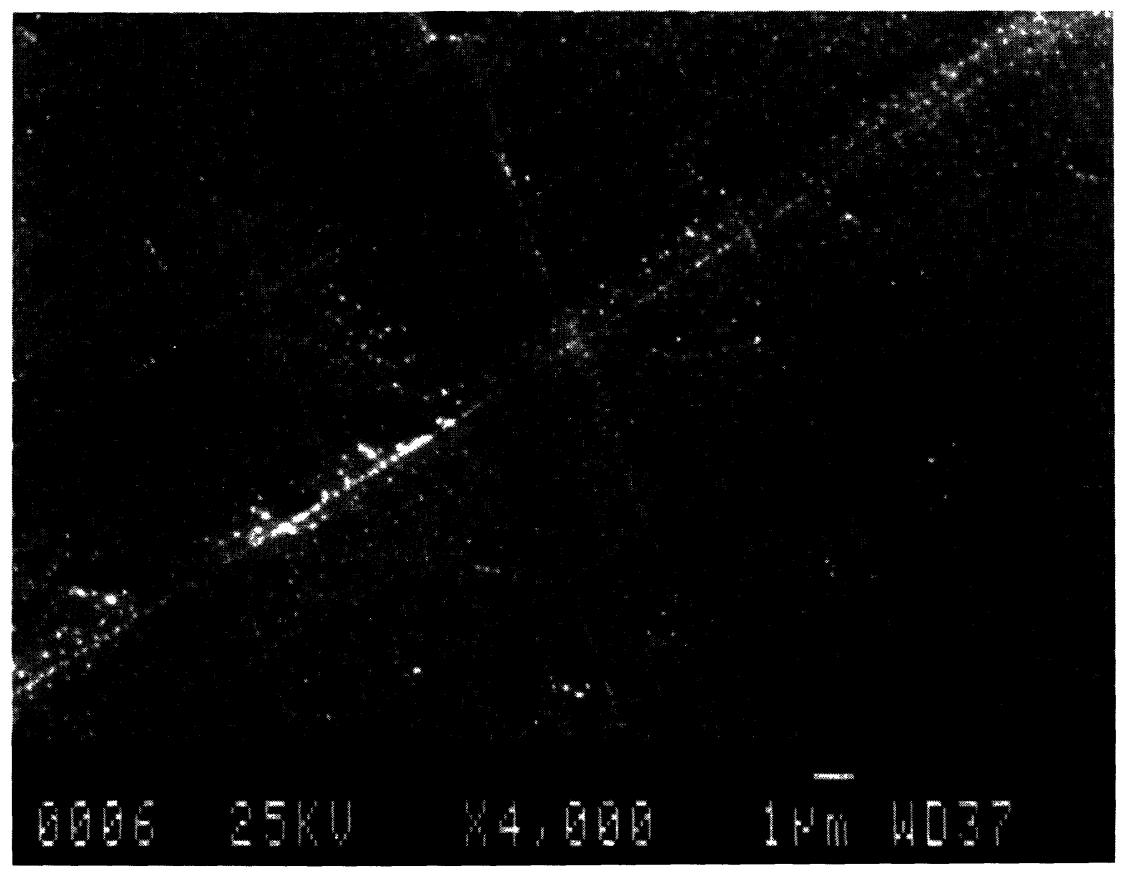

Fig. 5. - Slip traces on a Ge single crystal deformed at high temperature.

This procedure can sometimes yield unreasonable results, for instance when kinks still experience frictional stresses [6].

\section{Mobile dislocation densities.}

The last parameter interesting to measure to get the strain rate is the density of mobile dislocations. It is usually observed during in situ experiments that the mobile density is significantly lower than the total one. An easy way to measure it is to plot the density of dislocations which have moved during a time interval $\Delta t$ versus $\Delta t$ (the time "unit" being the duration of a video frame). The extrapolation to $\Delta t=0$ of this plot gives the instantaneous mobile dislocation density $\rho_{\mathrm{m}}$.

\section{Artifacts.}

Most artifacts encountered in in situ straining experiments have already been discussed [7]. The first condition to satisfy is that the thickness of the foil must be larger than the size of the studied structures (precipitate spacing, size of dislocation sources, ...). If not, specific mechanisms can operate, which are not found in the bulk, but which can nevertheless give interesting informations. For instance, in thin foils of superalloys, precipitates are often larger than the foil thickness. Precipitate shearing is then forced even at the beginning of the plastic deformation, instead of Orowan looping which is expected in the bulk at low strains. A careful analysis of dislocation recombinations as they come out from the precipitates is an useful way of checking the models proposed for the corresponding but slightly different mechanisms operating in the bulk at larger strains [8]. 
Surfaces can have a direct influence on dislocation motion through step formation which can slow down dislocations, mainly if a high modulus oxide layer prevents kinks from annihilating at the surface or through image forces which can lead to a decrease of dislocation density, and to preferential kink nucleation at surfaces. This last effect can considerably increase dislocation mobilities in the case of double kink mechanisms: velocity measurements on straight dislocations connecting the two surfaces without any bend or cusp should absolutely be discarded. Comparison with bulk materials of dislocation microstructures and if possible of measured parameters like activation energies (bulk straining) or dislocation velocities (e.g. X-Ray topography in semiconductors) is strongly recommended.

At high temperatures, evaporation of a particular chemical species can occur at surfaces, which leads to a modification of the composition of the material (e.g. $\mathrm{Li}$ in $\mathrm{Al} \mathrm{Li}$ alloys).

A second class of artifacts come from the electron beam. The previous limitations due to the foil thickness can be minimized by increasing the penetration of electrons. This is achieved through an increase of the acceleration voltage. But radiation damage occurs for beam energies larger than a threshold ranging between 300 and $400 \mathrm{kV}$ for most common metals. The compromise between penetration and radiation damage is illustrated in figure 6 .

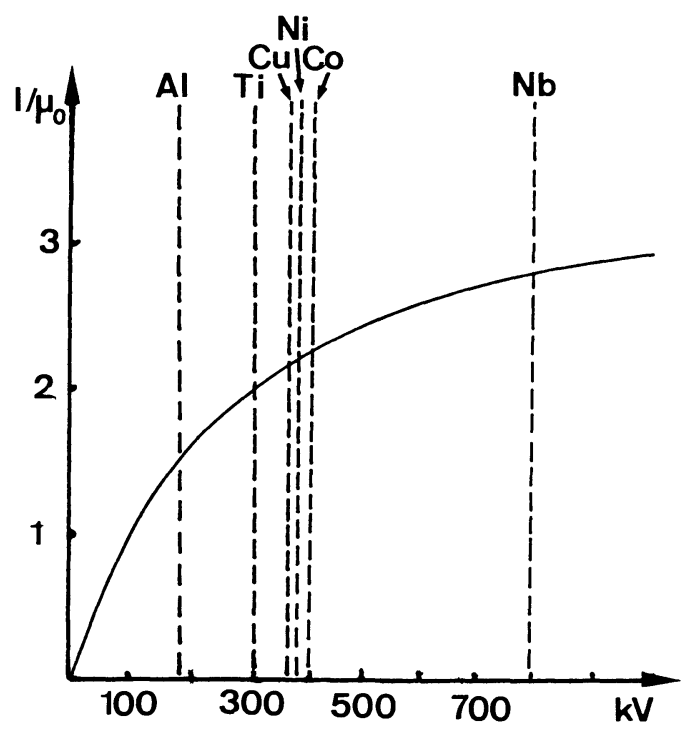

Fig. 6. - Penetration $\mu_{0}$ vs accelerating voltage, where $\mu_{0}$ is the damping coefficient of beam intensity $I$ defined by $I=I_{0} \exp \left(-\mu_{0} t\right)$. Radiation thresholds for various metals are indicated.

Local heating by electrons can also activate dislocation motion, but this effect is usually small. On the opposite, in specific materials like compound semiconductors, beam electrons create an appreciable quantity of electron-hole pairs, whose recombination at dislocations considerably helps dislocation motion [4]. The dislocation velocity in the dark is obtained by extrapolating at zero beam intensity the various velocities measured at different beam intensities. 


\section{Conclusion.}

We have shown that reliable quantitative information can be obtained from in situ experiments. External load and local stresses must be determined separately, the latter being estimated through the curvature radii of dislocations. Local strains can be reached by measuring the number of dislocations scanning the observed area or the corresponding step heights at the hole edge. Measurements of dislocation velocities yield a determination of the associated activation energies through techniques depending on the type of material. These informations can be considered as reliable provided some precautions concerning foil thickness and accelerating voltage, the best compromise for most common metals being a voltage between 300 and $400 \mathrm{kV}$.

\section{References}

[1] Pelissier J. and Debrenne P., this conference.

[2] Coujou A., Acta. Metall. 38 (1990) p. 825.

[3] GotTsChalk H., J. Phys. C4 (1983) 44.

[4] Louchet F., Pélissier J., Vanderschaeve G., Levade C., Peyrade J.P. and Caillard D., this conference.

[5] Couret A., Crestou J., Clément N., Coujou A., Farenc S. and Caillard D., this conference.

[6] LOUCHET F., TRIKI A. and PÉLISSIER J., this conference.

[7] MARTIN J.L. and KUBIN L.K., Ultramicroscopy 3 (1978) 215.

[8] Courbon J., Louchet F., Ignat M., Pélissier J. and Debrenne P., Phil. Mag. Letters 63, nº 2 (1991) 73-78. 